19 Revue d'histoire du XIXe siècle

Société d'histoire de la révolution de 1848 et des

révolutions du XIXe siècle

49 | 2014

1814-1815. Expériences de la discontinuité

\title{
Introduction : le désordre du temps
}

Emmanuel Fureix et Judith Lyon-Caen

URL : http://journals.openedition.org/rh19/4740

DOI : $10.4000 /$ rh 19.4740

ISSN : $1777-5329$

Éditeur

La Société de 1848

Édition imprimée

Date de publication : 1 décembre 2014

Pagination : 7-17

ISSN : 1265-1354

Référence électronique

Emmanuel Fureix et Judith Lyon-Caen, «Introduction : le désordre du temps », Revue d'histoire du XIXe siècle [En ligne], 49 | 2014, mis en ligne le 19 janvier 2015, consulté le 02 mai 2019. URL : http:// journals.openedition.org/rh19/4740 ; DOI : 10.4000/rh19.4740 


\section{EMMANUEL FUREIX, JUDITH LYON-CAEN}

\section{Introduction : le désordre du temps}

Les commémorations sont rarement propices aux bouleversements historiographiques. Totalement éclipsé par l'éclatant centenaire de la Grande Guerre, le bicentenaire des tumultueuses années 1814-1815 n'a guère provoqué de vagues, encore moins de controverses intellectuelles. On relève quelques commémorations ponctuelles, pour l'essentiel sous un angle napoléonien, et de rares colloques en France ${ }^{1}$, des manifestations plus vivantes ailleurs en Europe, notamment en Allemagne et en Suisse, où l'effondrement napoléonien coïncide avec une renaissance nationale ${ }^{2}$. Cette atonie française en dit assez long sur l'état d'une mémoire atrophiée ou antiquaire, mais aussi d'une historiographie très inégalement renouvelée.

Rappelons tout de même, de manière plus positive, quelques fronts pionniers ouverts depuis une trentaine d'années à propos de l'histoire des Restaurations. Le regain d'intérêt pour le libéralisme, dans les années 1980, permit de lire la Restauration comme un moment d'apprentissage (certes modeste) du parlementarisme, et d'affirmation d'une singularité libérale française, particulièrement autour des doctrinaires ${ }^{3}$. L'histoire de l'État et des techniques de gouvernement a souligné l'importance de ce moment dans la culture de l'enquête et l'observation de "l'état des esprits"4, mais aussi dans la perception fine du territoire et la modernisation de l'économie centrée sur la nation,

1. En particulier deux colloques organisés à Clermont-Ferrand et Paris par Jean-Claude Caron et Jean-Philippe Luis, Rien appris, rien oublié? Restaurations dans l'Europe post-napoléonienne (7 au 8 octobre 2013 et $12-13$ juin 2014), ainsi que plusieurs colloques relatifs à la campagne de France (à Lyon et à Troyes), et sur l'occupation russe (Les Russes en France en 1814, des faits, des imaginaires et des mémoires, Université Paris 1, les 16 et 17 octobre 2014).

2. Cf. notamment la célébration du bicentenaire de l'entrée de Genève et du canton de Neuchâtel dans la Confédération suisse (respectivement http://www.ge200.ch et http://www.bicentenaire2014. ch).

3. Pierre Rosanvallon, La monarchie impossible. Les Chartes de 1814 et de 1830, Paris, Fayard, 1994; Alain Laquièze, Les origines du régime parlementaire en France, 1814-1848, Paris, PUF, 2002; Pierre Rosanvallon, Le moment Guizot, Paris, Gallimard, 1985; Lucien Jaume, L'individu effacé ou le paradoxe du libéralisme français, Paris, Fayard, 1997.

4. Pierre Karila-Cohen, L'état des esprits. L'invention de l'enquête politique en France (1814-1848), Rennes, Presses universitaires de Rennes, 2008. 
"à mi-chemin de Colbert et de Smith ${ }^{5}$. Notre connaissance des relations sociales dans le monde postrévolutionnaire s'est aussi affinée, notamment à la lumière d'importants travaux sur la philanthropie, ses réseaux et ses impulsions réformatrices, notamment en matière d'éducation, d'assistance et d'enfermement ${ }^{6}$. Le cultural turn, aux Etats-Unis comme en France, a permis de relire en profondeur l'histoire politique de la période. L'espace public sous la Restauration est désormais irréductible à ses formes instituées (les Chambres, instances de délibération, et la presse, relégitimée par la Charte) : il s'étend à des pratiques informelles qui ont puissamment participé à la politisation des individus. Des banquets ${ }^{7}$ aux enterrements protestataires ${ }^{8}$, des représentations théâtrales ${ }^{9}$ aux répertoires de chansons ${ }^{10}$ et de poésies ${ }^{11}$, des sérénades aux charivaris, des rumeurs ${ }^{12}$ aux cris séditieux ${ }^{13}$, des pétitions ${ }^{14}$ aux souscriptions publiques ${ }^{15}$, un espace informel du politique s' est cristallisé, privilégiant la visibilité des opinions à leur formation procédurale, l'imaginaire sensible à la rationalité critique. Des approches transnationales ou connectées suggèrent de saisir le régime des Bourbons dans le mouvement européen des restaurations monarchiques ${ }^{16}$, le libéralisme dans une «internationale» active

5. Francis Démier, "Relecture de la Restauration", Revue d'histoire du XIXe siècle, 1994 ; cf. aussi sa récente synthèse La France de la Restauration (1814-1830), Paris, Gallimard / Folio Histoire, 2012.

6. Catherine Duprat, Usage et pratiques de la philanthropie. Pauvreté, action sociale et lien social, à Paris, au cours du premier XIX'e siècle, Paris, Comité d'histoire de la sécurité sociale, 2 volumes, 19961997 ; Jean-Luc Chappey, Carole Christen et Igor Moullier [dir.], Joseph-Marie de Gérando (17721842). Connaître et réformer la société, Rennes, Presses universitaires de Rennes, 2014.

7. Vincent Robert, Le temps des banquets. Politique et symbolique d'une génération (1818-1848), Paris, Publications de la Sorbonne, 2010.

8. Emmanuel Fureix, "Un rituel d'opposition sous la Restauration : les funérailles libérales à Paris (1820-1830)", Genèses. Sciences sociales et histoire, $n^{\circ}$ 46, mars 2002, p. 77-100; La France des larmes. Deuils politiques à l'âge romantique (1814-1840), Seyssel, Champ Vallon, 2009.

9. Sheryl Kroen, Politics and Theater. The Crisis of Legitimacy in Restoration France, Berkeley, University of California Press, 2000; Corinne Legoy, "Sous la plume du pouvoir, le public de théâtre entre 1815 et 1830 : l'embarrassant miroir d'une nation souveraine ", Parlement[s]. Revue d'histoire politique, 2012/3, p. 95-108.

10. Philippe Darriulat, La Muse du peuple. Chansons politiques et sociales en France, Rennes, Presses universitaires de Rennes, 2011 ; Sophie-Anne Leterrier, Béranger. Des chansons pour un peuple citoyen, Rennes, Presses universitaires de Rennes, 2013.

11. Corinne Legoy, L'Enthousiasme désenchanté. Éloge du pouvoir sous la Restauration, Paris, Société des Études robespierristes, 2010.

12. François Ploux, De bouche à oreille, naissance et propagation des rumeurs dans la France du XIX $X^{e}$ siècle, Collection historique, Paris, Aubier, 2003.

13. Gilles Malandain, L'introuvable complot. Attentat, enquête et rumeur dans la France de la Restauration, Paris, Éditions de l'EHESS, 2011, p. 269-281; Natalie Petiteau, "Violence verbale et délit politique, 1800-1830", Revue d'histoire du XIX'e siècle, n 36, 2008/1, p. 75-90.

14. Benoît Agnès, L’appel au pouvoir : essai sur le pétitionnement auprès des chambres législatives et électives en France et au Royaume-Uni entre 1814 et 1848, thèse de doctorat d'histoire sous la dir. de Christophe Charle, Université de Paris 1, 2009.

15. Emmanuel Fureix, "Souscrire pour les morts. Un don politique sous la Restauration et la monarchie de Juillet», Hypothèses, 2001/1, p. 275-285.

16. Mark Jarrett, The Congress of Vienna and its Legacy, Londres, Tauris, 2013; David Laven et Lucy Riall [dir.], Napoleon's Legacy. Problems of Goverment in Restoration Europe, Berg, Oxford-New York 2000. 
dans les années $1820^{17}$, l'héroïsme et la gloire dans un monde post-napoléonien désenchanté ${ }^{18}$, etc.

Ces historiographies ont en commun d'appréhender la période selon des découpages de régimes, isolant le plus souvent la Restauration, ou les monarchies constitutionnelles. C'est un autre regard que propose ce numéro, autour du singulier moment 1814-1815, englobant la chute de l'Empire, la première Restauration, les Cent-Jours et la deuxième Restauration. Il s'agit de penser ensemble ces deux années, sous l'angle de la discontinuité, et du point de vue de leurs acteurs et de leurs expériences. Et ainsi de rompre, autant que faire se peut, avec un récit linéaire ou univoque - tantôt napoléonien, tantôt bourbonien. Des écrits divers, dans le temps même de l'événement ou dans un immédiat après-coup, ont pris en charge le changement pour lui donner un sens, une direction, dans un cadre conflictuel où le sens de ce qui advenait constituait un enjeu majeur. Ces écrits, parce qu'ils font trace, modèlent fortement toute appréhension de ce passé conflictuel, et imposent à l'historien un écran qu'il est difficile de traverser.

Les ingrédients du chaos hexagonal de 1814-1815 sont bien connus : l'écroulement de la société impériale ${ }^{19}$, le traumatisme des défaites successives $^{20}$, deux invasions-occupations et leurs cortèges de violences ${ }^{21}$, la rétractation des frontières nationales, la démobilisation de l'armée napoléonienne ${ }^{22}$ et l'effondrement de son grand récit glorieux, la dilution de l'autorité dans une vacance du pouvoir ou la superposition de pouvoirs concurrents ${ }^{23}$, l'expérience de quatre régimes politiques qui sont des ré-instaurations plus que des restaurations, des épurations d'ampleur variable ${ }^{24}$, la recrudescence du phénomène rébellionnaire ${ }^{25}$, la réouverture de cycles vindicatoires initiés sous la Révolution ${ }^{26}$, des tentatives, souvent inabouties, de pardon, d'amnésie, d'amnistie et de réconciliation ${ }^{27}$. La versatilité des élites, les serments prêtés,

17. Maurizio Isabella, Risorgimento in Exile: Italian Émigrés and the Liberal International in the Post-Napoleonic Era, New York, Oxford University Press, 2009.

18. Luigi Mascilli Migliorini, Le mythe du héros. France et Italie après la chute de Napoléon, Paris, Nouveau Monde Éditions, 2003; Daniel Fabre, "L'atelier des héros», in La Fabrique des héros, Paris, Éditions de la Maison des sciences de l'homme, 1998, p. 233-318.

19. Sur cette dimension impériale de la société et du pouvoir napoléoniens, voir la synthèse novatrice d'Aurélien Lignereux, L'Empire des Français, 1799-1815, Paris, Seuil, 2012.

20. Jean-Marc Largeaud, Napoléon et Waterloo. La défaite glorieuse de 1815 à nos jours, Paris, Boutique de l'Histoire, 2006.

21. Jacques Hantraye, Les Cosaques aux Champs-Élysées, Paris, Belin, 2005.

22. Natalie Petiteau, Lendemains d'Empire. Les soldats de Napoléon dans la France du XIX' siècle, Paris, La Boutique de l'Histoire, 2003.

23. Marie-Cécile Thoral, «L'administration locale en temps de crise : le cas de l'Isère en 18141815", Annales historiques de la Révolution française, n 339, 2005, p. 117-135.

24. Jean Tulard, «Les épurations en 1814 et 1815 », Revue du Souvenir Napoléonien, nº 396, 1994, p. 4-21; Édouard Ebel, «D’une légalité à l'autre : gendarmerie et épurations, 1815-1816», Annales de Bretagne et des Pays de l'Ouest, 114-2, 2007, p. 147-158.

25. Aurélien Lignereux, La France rébellionnaire. Les résistances à la gendarmerie (1800-1859), Rennes, Presses universitaires de Rennes, 2008.

26. Lewis Gwynne, The Second Vendée. The Continuity of Counter-Revolution in the Department of the Gard, 1789-1815, Oxford, Clarendon Press, 1978.

27. Bettina Frederking, "Il ne faut pas être le roi de deux Peuples" : Strategies of National 
reniés et prêtés à nouveau ${ }^{28}$ qui nourrissent la figure de la girouette ${ }^{29}$ participent également au désordre des temps. Edgar Quinet, âgé de 11 ans en 1814 , lit rétrospectivement sa vie dans le sillage de cette crise de la temporalité, qui est aussi une crise du langage : «Ce qui me confondait, c'est que ce qui avait été honneur, vertu, devint subitement crime, infamie. [...] Or, je trouvais simple que les choses fussent différentes, mais je ne pouvais concevoir que les pensées, les paroles, et même les visages changeassent en même temps. L'impression de cette versatilité fut si grande, qu'elle s'est attachée à moi tout le reste de ma vie. [...] Je sentais la fragilité des choses, je sentais aussi celle des hommes ${ }^{30}$.

Comment penser la singularité de cette période? Le modèle, souvent invoqué, de la "transition", cher à la science politique - à travers la «transitologie» - impose généralement un modèle finaliste de démocratisation ou de pacification. Le concept de crise, qui renvoie à un délitement des relations de pouvoir, mais présuppose aussi un retour à la norme, ne paraît guère plus adapté. Aussi avons-nous privilégié celui de discontinuité.

La discontinuité n'est pas réductible à la rupture (ou aux ruptures) politique(s). Elle désigne une pluralité d'expériences, qui tiennent non seulement à l'incertitude du futur - c'est une évidence, en ces années de chassés croisés - mais plus généralement à la cassure du fil du temps. On peut interroger ces dérèglements à partir de la notion de "régime d'historicité», à condition d'articuler le questionnement sur l'expérience du temps à l'analyse des traces, multiples et discordantes, laissées par les acteurs du passé. Si l'on suit les propositions de Reinhart Koselleck, l'horizon d'attente, depuis la fin du XVIII ${ }^{\mathrm{e}}$ siècle, serait devenu plus ouvert, l'expérience collective du temps se faisant moins cyclique, moins répétitive. La brèche ouverte par la Révolution renforce cette tendance ${ }^{31}$. "L'expérience du temps, écrit François Hartog, est portée par le progrès et l'histoire devient processus. Elle s'écrit en allant du futur vers le passé ${ }^{32}$. Cette linéarité nouvelle se trouve mise à mal

Reconciliation in Restoration France', French History, 22, 4, déc. 2008, p. 446-468; Nathalie Scholtz, 'Past and Pathos. Symbolic Practices of Reconciliation during the French Restoration', History and Memory, Vol. 22, $\mathrm{n}^{\circ}$ 1, Spring/Summer 2010, p. 48-79; Noëlle Dauphin, "La loi d'amnistie du 12 janvier 1816 : volonté d'apaisement, mémoire de violence», in Jean-Claude Caron, Frédéric Chauvaud, Emmanuel Fureix et Jean-Noël Luc, [dir.], Entre violence et conciliation. La résolution des conflits sociopolitiques en Europe au XIXe siècle, Rennes, Presses universitaires de Rennes, 2008, p. 309324.

28. Jean-Yves Piboubès, Le serment politique en France, 1789-1870, thèse doctorat d'histoire sous la dir. d'Alain Corbin, Université Paris 1 Panthéon-Sorbonne, 2003.

29. Pierre Serna, La République des girouettes, 1789-1815 et au-delà. "L'extrême centre" : une anomalie politique, Seyssel, Champ Vallon, 2005, p. 143-304.

30. Edgar Quinet, Histoire de mes idées, in CEuvres complètes, tome 9, Paris, Pagnerre, 1857, p. 87.

31. Reinhart Koselleck, Le futur passé. Contribution à la sémantique des temps historiques, Paris, Éditions de l'EHESS, 1990.

32. François Hartog, "Historicité / régimes d'historicité», in Christian Delacroix, François Dosse et Patrick Garcia, Nicolas Offenstadt [dir.], Historiographies, tome 2, Concepts et débats, p. 767 et Régimes d'historicité. Présentisme et expériences du temps, Paris, Le Seuil, 2003. 
par l'expérience des années 1814-1815 : l'incertitude du présent et l'indétermination du futur se conjuguent à un possible retour vers le passé. L'incapacité des individus à vivre le présent, confrontés qu'ils sont au surgissement explosif d'un passé refoulé33, définit les contours d'une "crise du temps", telle que l'évoque Emmanuel de Waresquiel à propos des Cent-Jours ${ }^{34}$.

Différents rapports au temps entrent en concurrence, même à l'intérieur du groupe des "vainqueurs» du moment, et se redéfinissent à la lumière des expériences vécues dans le temps court. Ainsi les royalistes de 1814-1815 ne partagent-ils pas une vision commune du passé révolutionnaire et impérial, et en modifient leurs usages au fil des mois. Certains privilégient l'invocation à l'oubli, présente dans la Charte de 1814, et traduite juridiquement dans une amnistie ambiguë en janvier 1816. D'autres, à la lumière des Cent-Jours, réactualisent au contraire le spectre de la "guerre civile» révolutionnaire, préconisant l'expiation générale des "crimes» du passé, la stigmatisation des conventionnels régicides et des prêtres constitutionnels, mais aussi des "bonapartistes" notoires et des vétérans, et l'effacement spectaculaire des symboles du passé révolutionnaire et impérial ${ }^{35}$.

Le désaccord sur le sens présent du passé proche est lié à un désaccord plus général sur le sens des mots et sur la configuration de la souveraineté et de la légitimité. Ainsi, pour les uns, la Restauration de 1814 n'est pas une restauration mais une instauration, fragile et réversible : la légitimité de Louis XVIII ne dériverait que d'un appel de la nation via le Sénat conservateur, la souveraineté étant irrévocablement passée dans les mains de la nation en 1789. Pour les autres au contraire, la continuité dynastique et l'élection divine effacent la "parenthèse» révolutionnaire, l'enfoncent dans une temporalité abstraite. Parmi eux, certains imaginent un retour vers une souveraineté double du roi et de ses "corps naturels", la noblesse, les provinces, le clergé, etc. D'autres au contraire privilégient la seule souveraineté monarchique, les Chambres étant réduites, à leurs yeux, à une fonction de conseil du roi. De la même manière, pendant les Cent-Jours, les uns perçoivent le présent dans la continuité (cauchemardesque pour les royalistes) du passé impérial, quand les autres (certains «fédérés») y voient un retour de la révolution populaire et radicale, et d'autres encore un moment original, à nul autre pareil, mi-impérial mi-libéral. Plus généralement, les mots "honneur», «fidélité", "patrie», «liberté», sont devenus incompréhensibles à force d'être entendus en des sens contradictoires. Le désaccord va se nicher au cœur même des individus,

33. Sur l'idée de mémoire explosive, $c f$. Bronislaw Baczko, "La Pologne de Solidarité : une mémoire explosive", in Les imaginaires sociaux. Mémoires et espoirs collectifs, Paris, Payot, 1984, p. 185239.

34. Emmanuel de Waresquiel, L'histoire à rebrousse-poil. Les élites, la Restauration, la Révolution, Paris, Fayard, 2005, p. 152.

35. Cf. Emmanuel Fureix, «Une transmission discontinue. Présences sensibles de la Révolution française, de la Restauration aux années 1830 », in Sophie Wahnich [dir.], Histoire d'un trésor perdu. La transmission de l'événement révolutionnaire, 1789-2012, Paris, Les Prairies ordinaires, 2013, p. 149194. 
confrontés à leurs propres contradictions et à la langue glacée de la soumission à l'ordre des vainqueurs. De tout ceci découle un désordre des mots et des choses, les uns ne coïncidant pas avec les autres et recouvrant des temporalités différentes. L'actualité se trouve saturée d'anachronisme.

La notion de discontinuité ainsi que l'exploration des expériences de la discontinuité, permettent non seulement d'interroger ces désordres du temps, mais de faire ressurgir ce qui en a été ensuite oublié, effacé, enfoui, recouvert par les discours dominants. Michèle Riot-Sarcey, dans le sillage de Walter Benjamin, traque la discontinuité dans «un point de vue, une demande, un comportement singulier - et de ce fait peu représentatif d'un collectif - qui n'a eu, dans le temps postérieur à son avènement, aucun effet historique ${ }^{36}$. À titre d'exemple, un républicain démocrate comme Rigomer Bazin (17691818), s'efforce d'adapter au cadre de la Restauration une pensée de la République qui permette l'émancipation de l'opinion publique, une conscience civique forgée par l'éducation et par l'histoire, des droits de l'opposition, et même un horizon fédéral européen ${ }^{37}$. Discours inaudible dans la conjoncture du moment, qui conduit à l'assimiler à un dangereux jacobin illuminé. Ces pensées singulières, nourries d'un présent politique brouillé, ont été effacées par le cours de l'histoire.

Quel que soit le sens conféré au mot "discontinuité», l'historien se heurte aux "discordances des temps" (Christophe Charle) ${ }^{38}$ internes à une même société. En 1814-1815, cette pluralité des conceptions du temps est extrême. Les décalages des discours contemporains, leurs anachronismes conscients ou inconscients, le choc de la realpolitik rendent bien peu lisible l'ordre du temps. Ce moment pourrait bien être un laboratoire pour observer les liens entre des positions sociales et générationnelles et des manières de percevoir le temps. Liens à double sens, car les perceptions du temps ordonnent ellesmêmes de nouveaux découpages sociaux et politiques.

Dans cette perspective discontinuiste, ce numéro tente d'éclairer plusieurs pans de l'histoire sociale, politique et culturelle des années 1814-1815: l'ajustement des conduites individuelles face à un présent tumultueux; la gestion de l'autorité dans un régime d'incertitude; l'écriture de l'événement et dans l'événement.

Dans une conjoncture fluide, les positions et les fidélités des individus se recomposent à grande vitesse. Mais ces recompositions présupposent une information adéquate sur ce qui est en train d'advenir. Or, ainsi que le rap-

36. Michèle Riot-Sarcey, "Questionner l'histoire à "rebrousse poil" ", Espaces Temps, n 82-83, 2003, p. 17-27.

37. Pierre Serna, «Rigomer Bazin et la Restauration : penser la république dans la monarchie», Annales historiques de la Révolution française, $\mathrm{n}^{\circ} 325$, juillet-septembre 2001, p. 53-76.

38. Christophe Charle, Discordance des temps. Une brève histoire de la modernité, Paris, Armand Colin, 2011; Christophe Charle, "Autour de Discordance des temps : une brève histoire de la modernité», Vingtième Siècle. Revue d'histoire, n 117, 2013/1, p. 231-246 
pelle plus loin François Ploux, l'information entre à plusieurs reprises dans un régime d'incertitude, où les conjectures le disputent aux rumeurs. Le doute se traduit aussi dans une quête éperdue des signes visuels du changement, les couleurs et les symboles politiques devenant alors sursignifiés ${ }^{39}$. Sylvain Milbach, enquêtant aux frontières du territoire, dans une Savoie disputée, occupée, puis partagée, souligne également le besoin et l'attente frénétique de nouvelles - et l'espace laissé aux rumeurs par le desserrement administratif et politique. Cette incertitude, observe François Ploux, atteint son acmé au moment des transferts de pouvoir et de souveraineté, mais elle perdure d'autant plus que la légitimité des régimes ne cesse d'être remise en cause et que l'espace public s'ouvre à de nouveaux possibles. Les rumeurs se veulent quelquefois prédictives, voire prophétiques, pariant sur le renversement du cours présent de l'histoire.

En 1814-1815, le ralliement au pouvoir des vainqueurs pose de manière récurrente la question de l'appropriation des fonctions publiques, et plus généralement du travail des hommes sur les institutions en situation de crise. Pour les vaincus d'hier, et parfois des "obscurs", la Restauration représente une opportunité qu'ils s'efforcent de saisir. Soit directement par des usurpations de fonctions, avant que le pouvoir central n'intervienne dans la nomination de nouvelles autorités, soit indirectement, par appel au pouvoir sous forme d'adresse ou de sollicitation. Les remises en ordre biographiques et réinventions de passés sont alors légion : de faux "martyrs» de la Révolution pullulent dans les archives de la Restauration, tel Cugnet de Montarlot, ancien commissaire des guerres sous l'Empire, qui se dit en juillet 1814 «fils de chevalier de Saint-Louis, [...] du nombre de ceux qui ont été victimes de la Révolution $»^{40}$. Encore convient-il de maîtriser les justes codes de la reconversion.

S'ajuster au présent, c'est aussi, pour les élites en place, s'efforcer de résister au changement et maintenir leurs positions. Les girouettes, figures typiques de ces années 1814-1815, incarnent cette posture. Pour elles, la fidélité à l'ordre public peut légitimement transcender la fidélité à la personne du souverain, dans une logique d'extrême-centre que Pierre Serna a bien mise en exergue $^{41}$. Que la girouette soit devenue une sorte d'allégorie de l'Histoire en dit long sur le doute et le dégoût qui dominent l'imaginaire historique des contemporains. Qu'elle soit devenue, dans le même temps, un stigmate des réputations - via les dictionnaires de girouettes et autres écrits biographiques $^{42}$ - en dit tout aussi long sur la manière dont un espace public de la

39. Emmanuel Fureix, «Police des signes, ordre et désordre politiques en temps de crise (France, 1814-1816)", Histoire urbaine, 2015 (à paraître).

40. Cité par Laurent Nagy, D'une Terreur à l'autre. Théories du complot et nostalgie de l'Empire, 1815-1816, Paris, Vendémiaire, 2012, p. 119.

41. Pierre Serna, La République des girouettes, op. cit.

42. Jean-Luc Chappey, Ordres et désordres biographiques. Dictionnaires, listes de noms, réputation des Lumières à Wikipédia, Seyssel, Champ Vallon, 2013. 
notoriété et de l'honneur s'est mis en place depuis la fin du XVIII siècle $^{43}$. Par ailleurs, les luttes pour le pouvoir deviennent si acérées que des campagnes ad hominem cherchent à dissuader de potentielles girouettes de se reconvertir dans le nouvel ordre politique : le cas de Cambacérès, victime d'une quasi mise à mort graphique sous la première Restauration, est à cet égard remarquable ${ }^{44}$. La violence de la critique des trahisons réelles ou supposées semble avoir été particulièrement forte en France : Matthijs Lok, en comparant les girouettes françaises et hollandaises, montre ici une plus grande acceptation de ces transfuges politiques du côté des Pays-Bas. Cette singulière violence française s'explique notamment par la compétition entre anciennes et nouvelles élites pour la maîtrise de l'espace public, élites dont la fusion s'est avérée particulièrement difficile ${ }^{45}$.

La girouette est une figure du temps court. Elle capte l'attention de l'historien vers les soubresauts et les volte-face. Mais la question de l'ajustement des positions s'est également jouée dans d'autres temporalités, selon les institutions concernées. L'enquête d'Olivier Tort montre ici combien l'épuration des magistrats sous la Restauration, plus sélective qu'on ne l'a dit, s'est aussi étirée dans la durée : au delà des nominations et des révocations spectaculaires, c'est le temps lui-même qui fait son œuvre, faisant planer une menace sourde sur ceux qui restaient en place mais permettant aux magistrats de «bonne volonté» de rentrer silencieusement dans le rang.

Deuxième terrain d'enquête possible : la gestion de l'autorité face au changement. Plus classique sans doute, il conduit d'abord à focaliser son attention sur les moments et les lieux de superposition des pouvoirs, en situation de frontière et dans toutes les zones où des souverainetés multiples en viennent à se superposer. L'invasion et l'occupation du territoire voient ainsi coexister non sans tension les pouvoirs civil, militaire et religieux. Les conflits de juridiction en cas de délits commis par des civils français sur des soldats d'occupation traduisent ces tensions ${ }^{46}$. Les conflits de légitimité, dans le Midi de la deuxième Restauration, entre les commissaires envoyés par le duc d'Angoulême et les agents désignés plus tardivement par Louis XVIII, sont également significatifs de ce moment politique.

Plus profondément, le temps discontinu et le régime d'incertitude rendent d'autant plus impérieuses la connaissance et la maîtrise de l'opinion publique, afin de mieux interpréter le bruissement du monde social. Ainsi, en 1814, le temps de l'enquête politique ne se dissocie pas de celui de

43. Antoine Lilti, Figures publiques. L'invention de la célébrité (1750-1850), Paris, Fayard, 2014.

44. Emmanuel Fureix, "La porte de derrière. Sodomie et incrimination politique : des caricatures contre Cambacérès (1814-1815)", Annales historiques de la Révolution française, 2010/3, p. 109-130.

45. Emmanuel de Waresquiel, L'histoire à rebrousse-poil..., op. cit.

46. Cf. Pierre-Baptiste Schmitt-Cadet, 1814-1818. Invasions et occupations : expériences et perceptions (Ain, Doubs, Nord), mémoire de master sous la dir. d'Emmanuel Fureix, Université ParisEst Créteil, 2013, p. 268 et sq. 
l'instauration du nouveau régime : des commissaires extraordinaires puis des délégués de police parcourent les départements à la recherche des "haines de partis». Puis le secrétaire général du ministère de l'Intérieur, le jeune Guizot, demande aux préfets d'établir une «statistique morale du royaume" sous la forme d'une "conversation familière $»^{47}$. Les élites savoyardes (nobiliaires et cléricales) sondent aussi et surtout invoquent l'opinion publique, via un "plébiscite occulte» orchestré à l'été 1815, ainsi que le montre Sylvain Milbach. Destiné à justifier la Restauration sarde, il est organisé dans une logique à la fois «nationalitaire» et notabiliaire.

Invoquer ou mesurer l'opinion publique ne saurait cependant suffire pour assurer la concorde civile. Dans ce volume, Pierre Triomphe envisage les stratégies déployées par les autorités pour sortir du cycle vindicatoire ouvert par la "Terreur blanche» de 1815 dans le Midi : un double compromis avec les élites et les classes populaires royalistes pour arrêter les violences, un maintien de l'ordre public par le contrôle des gardes nationales. Avec une tout autre méthode, d'esprit contre-factuel, Aurélien Lignereux démonte les mécanismes qui conduisirent à l'absence de massacre de masse dans l'Ouest rebelle de 1815. Il s'est agi pour lui de «saisir sur le vif le jeu croisé des dynamiques vindicatives et des mesures pacificatrices, en prenant au sérieux l'horizon imaginé par les contemporains». La restitution des armes enlevées aux patriotes, le voile judiciaire posé sur les exactions commises par les partisans des Bourbons, la distribution des places aux royalistes, constituent les principaux ingrédients de la pacification.

Interroger l'expérience de la discontinuité dans l'ajustement des attitudes ou l'exercice de l'autorité, c'est aussi se confronter à des sources écrites qui portent la marque de ces désordres, de ces discordances, qui les explicitent ou les taisent, les dramatisent ou les atténuent, mais aussi les instrumentalisent. Circulaires, enquêtes, documents policiers, judiciaires, administratifs ou écrits personnels (journaux, lettres), figurations littéraires (fictions, pièces de théâtre), articles de presse, écrits de circonstance (dictionnaires biographiques de girouettes, par exemple), pamphlets, placards, etc. : l'historien se trouve constamment aux prises avec une foule d'écrits produits dans le temps des événements, ou juste après. Toutes les contributions réunies ici montrent bien comment l'écrit a été constamment investi pour agir dans ces temps d'incertitude : des individus s'emparent de toutes les opportunités d'écriture possible pour se justifier, se mettre en avant, demander une place, disqualifier leurs ennemis; le récit des événements, leur mise en forme, leur interprétation historique, philosophique, religieuse parfois, sont l'enjeu de conflits incessants, de luttes de position; la maîtrise des informations, la qualification de leur nature (nouvelles «sûres» ou rumeurs), la traduction de leur

47. Pierre Karila-Cohen, op. cit. 
signification dans des gestes, des décisions, des prises de parti, se trouvent constamment au cour des actions individuelles et collectives. Envisager les expériences de la discontinuité en 1814-1815, saisir les conceptions discordantes et conflictuelles du temps, les écarts entre les expériences singulières et les discours - eux-mêmes fluctuants et pluriels - des dominants, traquer les sens perdus, enfouis, effacés, des événements conduit à ne pas considérer seulement ces écrits comme des «documents" ou des «sources" pour les historiens de cette période, mais bien à en prendre en compte l'épaisseur factuelle, à en apprécier la force agissante dans leurs contextes de production, de circulation et d'usage.

Olivier Bara propose ici une enquête sur un type d'écrits à la fois peu connu des études littéraires - parce que jugé «mineur» - et peu utilisé par les historiens, les innombrables pièces de circonstance jouées sur les scènes parisiennes entre la première et la deuxième Restauration. On saisit ici à la fois la question, épineuse, de l'ajustement des conduites des gens de théâtre aux changements politiques, les formes de symbolisation mobilisées pour rendre compte des événements, et les types d'action sur le temps que produisent ces pièces sans postérité, tant pour leurs auteurs et acteurs que pour leurs publics.

Certains de ces écrits trahissent la tentation, éprouvée par certains contemporains, de résorber la discontinuité par l'hypertrophie d'un passé imaginaire et monumental : pièces de théâtre et pièces de vers bourboniennes convergent ainsi dans la construction d'un «temps mythique», fondé sur "la continuité et la réitération", «la fusion instrumentalisée du présent, du passé et de l'avenir ${ }^{48}$. Matthijs Lok, en comparant des écrits de circonstance français et hollandais, introduit une nuance supplémentaire : quand la référence française à Henri IV plonge dans une temporalité effectivement mythologique, les allusions hollandaises à la révolte antiespagnole de la fin du XVI ${ }^{e}$ siècle ne figent pas le présent dans la simple répétition du passé.

Écrire la discontinuité : le document proposé au terme de ce dossier suggère une expérience de lecture, qui consiste à appréhender, dans son rythme et ses méandres, l'ajustement d'une écriture littéraro-journalistique aux mois de mars et d'avril 1814, quand le pouvoir impérial s'effondre. "L'Hermite de la Chaussée d'Antin", créé par Etienne de Jouy dans le feuilleton de $\mathrm{La}$ Gazette de France, meurt - fictivement - de l'entrée des troupes alliées dans Paris et disparait - pratiquement - des colonnes du journal pour permettre à son auteur de renaître sous le nouveau régime. Faire l'expérience de la lecture de ces feuilletons, c'est se confronter directement non pas seulement à une source sur la discontinuité du temps, mais bien à ce que la discontinuité fait à l'écriture (à ses conditions d'existence, comme à ses énoncés) et à ce que l'écrit fait de la discontinuité. 
La caricature reproduite en couverture concentre nombre de ces interrogations sur l'écriture de la discontinuitét ${ }^{4}$. Réalisée et diffusée au début de la deuxième Restauration, elle figure allégoriquement le Journal des débats, ancien Journal de l'Empire pendant les Cent-Jours, scindé en deux personnages : à droite un mousquetaire royaliste, revêtu des habits d'Arlequin, crie "Vive le roi! Vive le plus fort!»; à gauche un grenadier bonapartiste, portant un bonnet à cocarde tricolore et aigle impériale, crie «Vive la liberté! Vive l'empereur!». Le grenadier bonapartiste écrase du pied deux feuilles portant ces mots : "Vérité, patrie, justice», et sa main porte «fausses nouvelles, mensonges, dénonciations, vengeances, absurdités, partialités». La représentation des palinodies du Journal des débats renvoie à la fois au cynisme - la loi du plus fort, les «fausses nouvelles" - à la commedia dell'arte - l'Arlequin - et à un fait social plus général - la figure de la girouette. La caricature emprunte de fait son motif - un Janus bifrons - à une estampe de Dubois, «La girouette», produite pendant les Cent-Jours ${ }^{50}$.

Des girouettes politiques aux girouettes littéraires, des fausses nouvelles aux enquêtes politiques sur l'état des esprits, d'épurations en stratégies de maintien de l'ordre, des scènes de théâtre aux frontières disputées du territoire, ce numéro aura tenté une exploration des expériences de la discontinuité en 1814-1815, en se tenant davantage au niveau des acteurs que dans l'analyse des discours et des récits surplombants. L'historien hérite souvent, on l'a dit, des discours des vainqueurs. En se replongeant dans la cacophonie des années 1814-1815, en se laissant prendre à leur illisibilité, les contributeurs de ce numéro ont tenté de se déprendre des historiographies dominantes, héritées des luttes de ce temps, pour restituer des configurations effacées et des actions qui, tout en ayant été partie prenante des événements, se sont trouvées disqualifiées, exclues de la construction de leur sens. Par nature, l'enquête aura privilégié l'étude de cas sur la généralisation, le local sur le global. Le cadre national français est demeuré dominant : les articles de Matthijs Lok et de Sylvain Milbach suggèrent pourtant toutes les potentialités d'une approche comparatiste - dans une Europe où tous les bouleversement politiques sont étroitement connectés - et les apports des études transfrontalières et transnationales. Nous espérons que ce numéro suscitera d'autres jeux d'échelles.

Emmanuel Fureix est maître de conférences à l'Université Paris-Est Créteil, membre de l'Institut universitaire de France Judith Lyon-Caen est maitresse de conférences à l'Ecole des hautes études en sciences sociales

49. "Journal de l'Empire, ou des débats suivant les événements", Paris, chez tous les marchands de nouveautés, 1815. Dépôt légal le 29 juillet 1815 . British Museum, PPA45337.

50. «La girouette». Dépôt légal par Frédéric Dubois le 29 avril 1815. British Museum, PPA45230. 